\title{
Enterprise Credit Rating Based on Genetic Neural Network
}

\author{
Yulin Dua \\ Department of Finance, East China University of Political Science and Law, 201620, Shanghai
}

\begin{abstract}
The genetic BP algorithm is used to modify and optimize the connection weights and thresholds of the neural network, which solves the problem that BP neural network has slow convergence speed and may fall into local minimum to a certain extent. The accuracy of rating indicates that the genetic neural network method is very suitable for enterprise credit rating.
\end{abstract}

\section{Introduction}

Enterprise credit rating can provide fair credit information for economic management departments, financial institutions and investors to avoid risks, optimize investment and improve efficiency.

The traditional rating method based on statistics, expert system method and artificial neural network method. The idea of these methods is to find out the rules of classification and establish discriminant models from historical data, and then use them to classify new samples. BP Neural network provides a new method for credit rating. It is good at learning useful knowledge from input and output data, easy to implement parallel computing. It is expected to solve some problems which are difficult to be solved by traditional methods. But BP Neural networks have some shortcomings, such as local convergence, long training time and sensitivity to initial weights. In this paper, we adopt the method of adaptive learning rate and increasing momentum term (improved BP neural network algorithm) based on the application model of credit rating. First, we quantify the index system, as the input of the neural network model, through the learning algorithm to train continuously. When the error between the output and the actual output is less than a certain specified value, the training process stops, and then the weights and thresholds are determined to obtain the required network structure to get the rating results.

\section{Genetic algorithm BP neural network}

\subsection{BP neural network}

BP (Back Propagation) network is a multilayer feed forward neural network, the network training, adjust the training algorithm right threshold follows the error backpropagation method. Usually the BP neural network has 3 layers or 3 layers these neurons, including input layer, hidden layer and output layer, realize the connection between the upper and lower layer, and the same neuron connection. The input layer neurons and neurons in the hidden layer and hidden layer between neurons and neurons in the output layer is the network weights, the connection strength between two neurons in the hidden layer.

The steps of BP neural network are as follows:

(1) Selecting hidden layer numbers.

Lippman had proved no matter how complex a function mapping is, it is enough to meet the neural network needs. We will use a single hidden layer structure to build a BP neural network model.

(2) Determining the number of neurons in the hidden layer.

The network with different hidden layer neurons is trained by the same sample set until the weight is unchanged. The number of hidden layer neurons is determined by the principle of minimum error. we will use 6 as the number of neurons in the hidden layer.

(3) Transfer function.

Because there is no linear relationship between the output vector and the input vector set $X=\left(X_{1}, X_{2}, \mathrm{~L}, X_{16}\right)$, and considering that the neural transfer function of BP neural network should be differentiable everywhere, sigmoid function is defined as the transfer function:

$$
f(x)=\frac{1}{1+e^{-x}}
$$

(4) Training times.

After training, the test error at this time should be extracted, and each time the test error is extracted, the weight of the moment should be retained.

(5) Output layer.

The output layer of this model only needs to reflect the credit rating of the enterprise, so we set only one output layer node.

\footnotetext{
${ }^{\mathrm{a}}$ Corresponding author: tongjihz@sina.com
} 


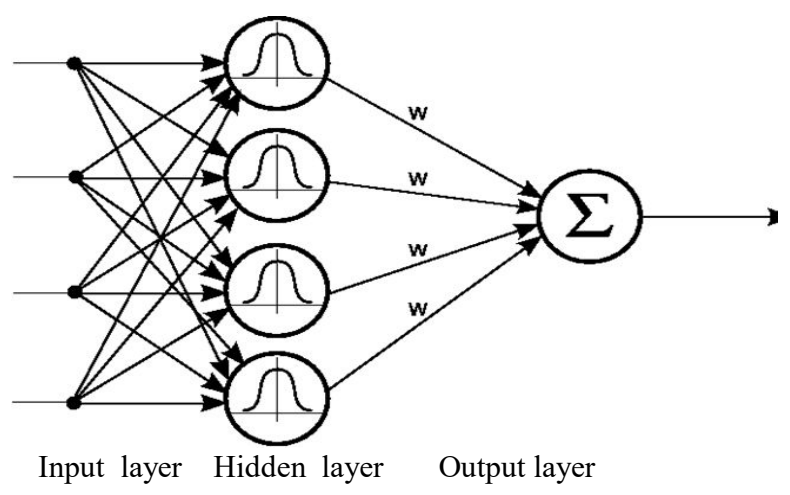

Figure 1. The structure of BP neural network

(6) Establishing models

We designs a three-layer neural network model to simulate the enterprises credit rating. The model has 16 input layer nodes, i.e. input vectors $X=\left(X_{1}, X_{2}, \mathrm{~L}, X_{16}\right)$, which correspond to 16 indexes in the credit rating system of enterprises, and the hidden layer of the model has six nodes, i.e. hidden layer vectors. The weights between the input layer and the hidden layer and between the hidden layer node $Y=\left(Y_{1}, Y_{2}, \mathrm{~L}, Y_{k}\right)$ and the output layer node are expressed by vectors and vectors respectively.

$$
\text { Output }=\sum_{i=1}^{16} f\left(w_{i j} y_{i}\right)
$$

$f(x)$ is the Sigmoid transfer function.

\subsection{GABP neural network}

Genetic algorithm (GA) is a mathematical model designed to imitate the rules of natural selection. GABP process is divided into four parts: initialization, selection, crossover and mutation. The algorithm does not fall into local minimum when searching for the optimal value, and is a common method for global optimization. BP algorithm uses gradient method to search the optimal solution, which leads to its weak ability to search the optimal solution, easy to trap in the local optimal solution. The advantage of genetic algorithm is that it can quickly find the global optimal solution. The idea of combining the two is that binary coding is used to represent the weights randomly given by the network as the initial group, then the output value is calculated by using the weights of each group, then the fitness is calculated according to the output value, the probability of the network being selected is determined according to the fitness,so the process of genetic variation between the weights of the two groups is obtained.

The steps of using legacy algorithm to improve the neural network model are as follows:

(1) Generates a binary string set, which represents the set of connection weights. The relationship between binary weight and real weight is:

$\omega_{t}(i, j, k)=\omega_{\min }(i, j, k)+b(\mathrm{t})\left(\omega_{\max }(i, j, k)-\omega_{\min }(i, j, k)\right)$
Where $b \quad(\mathrm{t})$ represents the binary string, $\omega_{\max }(i, j, k)$ and $\omega_{\min }(i, j, k)$ is the maximum and minimum of the join weight.

(2) An initial group of connection options is randomly generated and the binary connection weights are translated into real-valued weights.

(3) The output results can be obtained by combining the connection weights and input variables of real numbers. According to the size of the fitness to determine the probability of selecting the network, its probability value is:

$$
p=\frac{f_{i}}{\sum_{i} f_{i}}
$$

$P$ represents the network i-th in evolution of the selected probability, $f_{i}$ represents its fitness.

(4) According to the above probability value, two networks are randomly selected, and the two networks are crossed by a certain probability to produce a new network. Then the new network is mutated according to the probability of $P_{m}$, so the next generation network is obtained.

(5) Repeat (2) (4) until the required error is reached.

\section{Empirical Results and Analysis}

\subsection{Data}

We select 100 listed companies in China in 2017 as training sample and test sample. Training sample include 50 companies, including 30 ST companies, 20 non-loss companies; testing sample includes 50 companies, including 30 ST companies, 20 non-loss companies, The financial index of these companies are chosen as follows.

Table1. Finacial Index Selected

\begin{tabular}{|c|c|}
\hline Solvency & $\begin{array}{c}\text { Asset liability ratio } \\
\text { Flow rate } \\
\text { Speed ratio }\end{array}$ \\
\hline Operational capability & $\begin{array}{c}\text { Accounts receivable turnover rate } \\
\text { Turnover of current assets } \\
\text { Turnover of total assets }\end{array}$ \\
\hline Profitability & $\begin{array}{c}\text { Return on assets } \\
\text { Gross profit margin }\end{array}$ \\
\hline Development capacity & $\begin{array}{c}\text { Asset growth rate } \\
\text { Annual profit growth rate } \\
\text { Market share growth rate }\end{array}$ \\
\hline Economic strength & Asset rate quantification \\
& Cash flow rate of return \\
& total assets \\
\hline operating efficiency & $\begin{array}{c}\text { Sales profit margin } \\
\text { Net asset interest rate }\end{array}$ \\
\hline
\end{tabular}


In order to eliminate different financial index between dimension and numerical value differences in learning errors of each factor, we standardize the financial index through the equation:

$$
x_{i}=\frac{x_{t}-x_{\min }}{x_{\max }-x_{\min }} .
$$

$x_{t}$ is the original data, $x_{\max }, x_{\min }$ is the minimum and maximum of $x_{t}, x_{i} \in[0,1]$.

We can restore to the original data $x_{t}$ through:

$$
x_{t}=x_{\min }+x_{i}\left(x_{\max }-x_{\min }\right) \text {. }
$$

The results below are obtained by Matlab2015.

\subsection{Model Training}

The number of hidden layer nodes is basically based on experience. We calculate the relationship between the number of nodes in different hidden layers and the error, and find that the error is the smallest when choosing 6 layers.

Table 2. Error of different Hidden layers

\begin{tabular}{|c|c|}
\hline Hidden layer number & Error \\
\hline 4 & 0.1459 \\
\hline 5 & 0.1387 \\
\hline 6 & 0.1341 \\
\hline 7 & 0.1355 \\
\hline 8 & 0.1398 \\
\hline 9 & 0.1423 \\
\hline 10 & 0.1438 \\
\hline 11 & 0.1459 \\
\hline 12 & 0.1489 \\
\hline
\end{tabular}

The neural network optimized by genetic algorithm achieves the training error faster than the training requirement error.

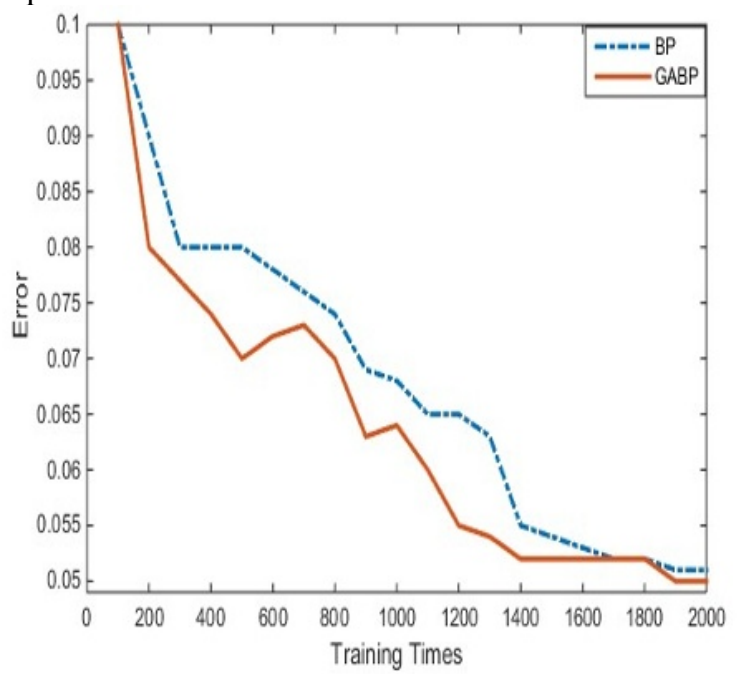

Figure 2. Result of BP Neural Network model

\subsection{Model Testing}

After the training, the financial indexedata of 50 listed enterprises are input into BP neural network and genetic algorithm to optimize BP neural network model to test. At the same time, the results of the network output and the real rating results are compared and statistically analyzed. The model test results are shown in Table 3.

Table 3. The rating test results of models

\begin{tabular}{llll}
\hline & $\begin{array}{l}\text { Expert } \\
\text { rating }\end{array}$ & $\begin{array}{l}\text { BP } \\
\text { rating }\end{array}$ & $\begin{array}{l}\text { GBP } \\
\text { rating }\end{array}$ \\
\hline 1 & AA & AA & AA \\
2 & A & A & A \\
3 & BBB & BBB & BBB \\
4 & BB & BBB & BB \\
5 & C & C & C \\
6 & AAA & AAA & AA \\
7 & AAA & AA & AAA \\
8 & BBB & BBB & BBB \\
9 & BB & BB & BB \\
10 & A & A & A \\
11 & CC & C & CC \\
12 & AAA & AAA & AAA \\
13 & AA & AA & AA \\
14 & AAA & AAA & AA \\
15 & AA & AA & AA \\
16 & BB & B & BB \\
17 & AA & AA & AA \\
18 & C & C & C \\
19 & BBB & BB & BBB \\
20 & AA & AA & AA \\
\hline
\end{tabular}

From the table, we can see BP results differ from expert results by five, while GABP results differ from expert results by only two companies. It shows that the result of GABP model is more accurate.

\section{Conclusions}

In this paper, we use genetic algorithm to optimize the weights and thresholds of BP neural network model, and establishes an enterprise credit rating model based on genetic neural network. The results show that the genetic algorithm credit rating model can be used in enterprise credit rating.

\section{References}

1. Li $\mathrm{W}$, Yan $T$, Liang $Y$ J. Pressure Prediction Technology of the Deep Strata Based on BP Neural Network[]]. Advanced Materials Research, 2010, 143-144(2):28-31

2. Jiang G. Research on Credit Rating Method Based on BP NN[C]// International Conference on Service Systems and Service Management. IEEE, 2007:1-4 
3. Jiang L. The Applications of GA-BP Neural Networks in Option Direction for Catalytic Agent[J]. Applied Mechanics \& Materials, 2014, 510:45-50.

4. Wang $Y Z$, Lei $X$, Chen $X$ S, et al. Large power customer credit rating based on BP neural network[]]. Power Demand Side Management, 2015.
5. Zhang $\mathrm{H}$, Ding $\mathrm{Y}$. Enterprise credit rating model based on BP neural networks[]]. Journal of Shanghai Maritime University, 2007.

6. Zheng $\mathrm{H}$. Optimization and Implementation of GaBP Algorithm[J]. Journal of Longyan University, 2015. 